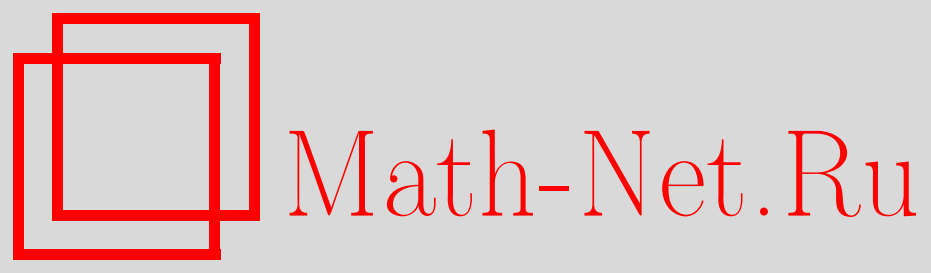

И. Кушнир, Бипедальные равновеликости, Квант, 2020, номер 6, 25-28

DOI: https://doi.org/10.4213/kvant20200603

Использование Общероссийского математического портала Math-Net.Ru подразумевает, что вы прочитали и согласны с пользовательским соглашением http://www.mathnet.ru/rus/agreement

Параметры загрузки:

IP : 3.85 .73 .92

26 апреля 2023 г., $17: 05: 55$

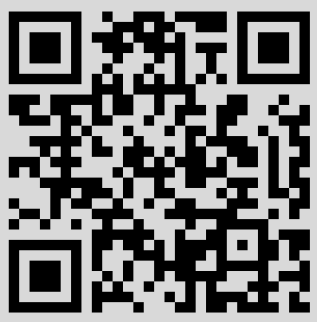


Примечание. Для малого угла $\varphi$ справедливы приближенные формулы $\cos \varphi \approx 1 u \sin \varphi \approx \varphi$.

На рисунке 2 показан ход двух параллельных лучей, составляющих известный угол $\alpha$ с горизонтальной нормалью к поверхности сферы. В точке $A$ на пересечении

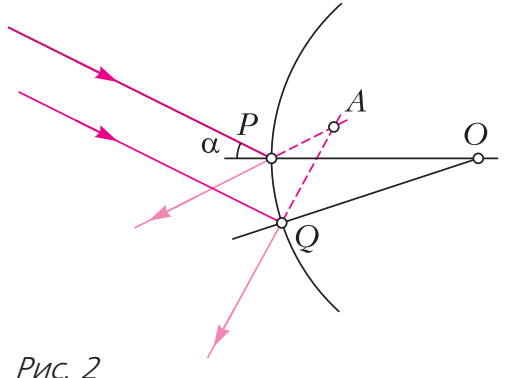

продолжений отраженных лучей будет наблюдаться изображение Солнца. Если угол между радиусами, проведенными в точки $P$ и $Q$, равен $\angle P O Q=\Delta$, то угол между продолжениями отраженных лучей равен $\angle P A Q=2 \Delta$, поскольку при повороте зеркала на угол $\Delta$ отраженный луч поворачивается на $2 \Delta$. Угол $\Delta$ определяется размером зрачка и, очевидно, мал. Другие углы равны, оответственно,

$$
\angle A P O=\alpha, \angle A Q P=\frac{\pi}{2}-\alpha-\Delta \approx \frac{\pi}{2}-\alpha .
$$

Запишем теорему синусов для треугольника $P A Q$ и, учитывая что $2 \Delta-$ малый угол, получим равенство

$$
P A=\frac{P Q}{2 \Delta} \cos \alpha .
$$

\section{Бипедальные равновеликости}

\section{И.КУШНИР}

В этой статье мы расскажем о нескольких геометрических «приключениях» и, в частности, решим задачу М2590. В них появятся равновеликие фигуры в конструкциях с перпендикулярами, проведенными из точки, лежащей на стороне треугольника, к двум его другим сторонам. Такие перпендикуляры мы будем называть «бипедалями».

Приключение первое. Пусть в остро-

DOI: https://doi.org/10.4213/kvant20200603
Отсюда, поскольку справедливо приближенное соотношение $P Q \approx R \Delta$, находим

$$
P A=\frac{R \cos \alpha}{2} .
$$

Можно убедиться в том, что это уравнение задает полуокружность радиусом $0,25 R$, центр которой располагается на отрезке $O P$ на расстоянии $0,75 R$ от точки $O$. При изменении угла $\alpha$ точка $A$ сдвигается по этой окружности. Угловой размер Солнца равен $\varphi$, поэтому угол падения пучков параллельных лучей от Солнца изменяется в пределах от $\alpha$ до $\alpha+\varphi$. Пусть лучам, падающим под углом $\alpha$, соответствует точка $A$, а лучам, падающим под углом $\alpha+\varphi,-$ точка $A_{1}$ (рис.3). Характерный размер

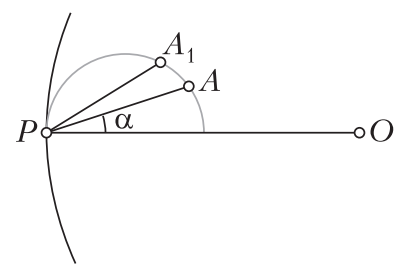

Pnc. 3

изображения Солнца можно считать равным высоте треугольника $P A_{1} A$, опущенной из вершины $A_{1}$. В первом приближении эта высота равна

$$
\Delta L=\frac{R \cos \alpha}{2} \varphi .
$$

А.Бъчков, П.Крюков угольном треугольнике $A B C$ проведена высота $A H_{1}$, из $H_{1}$ проведены бипедали $H_{1} M_{H}$ и $H_{1} N_{H}$ (рис.1).

Пусть $O$ - центр описанной окружности треугольника $A B C$. Выдающийся геометр 3.А.Скопец рассматривал условие принадлежности О прямой «посадил» точку $O$ на отрезок $M_{H} N_{H}$ ! Оказывается, при вы-

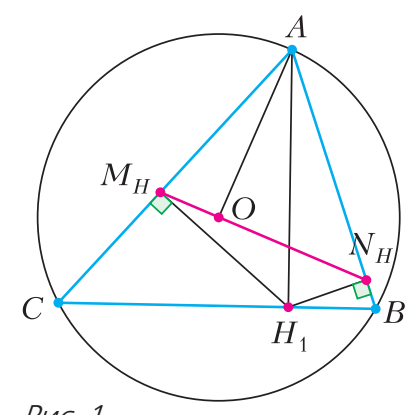

Рис. 1 
полнении этого условия отрезок будет «равноделящей», т.е. разделит площадь треугольника пополам. Итак,

Задача 1. В остроугольном треугольни ке АВС известно, что точка О лежит на отрезке $M_{H} N_{H}$. Докажите, что отрезок $M_{H} N_{H}$ делит площадь треугольника АВС пополам.

Вначале приведем схему «формульного» решения задачи.

Понадобятся следующие утверждения для произвольного треугольника $A B C$, которые предлагаем читателю доказать самостоятельно:

1. $O A \perp M_{H} N_{H}$;

2. $M_{H} N_{H}=\frac{S}{R}$,

где $S$ - площадь треугольника $A B C, R-$ радиус окружности, описанной около треугольника $A B C$.

Из утверждений 1 и 2 легко следует решение задачи. Поскольку $O A \perp M_{H} N_{H}$, то в треугольнике $A M_{H} N_{H}$ отрезок $O A$ будет высотой, а длина стороны $M_{H} N_{H}$ равна $\frac{S}{R}$, значит,

$$
S_{A M_{H} N_{H}}=\frac{1}{2} R \cdot \frac{S}{R}=\frac{1}{2} S .
$$

Поэтому площади треугольника $A M_{H} N_{H}$ и четырехугольника $M_{H} C B N_{H}$ равны, а $M_{H} N_{H}-$ равноделящая.

Задача имеет и чисто геометрическое решение.

Проведем диаметр $A A_{1}$ (рис.2). Поскольку $M_{H} O$ - медиана треугольника $A M_{H} A_{1}$, то $S_{A M_{H} O}=S_{A_{1} M_{H} O}$. Аналогично, $S_{A N_{H} O}=$ $=S_{A_{1} N_{H} O}$. Отсюда $S_{A M_{H} N_{H}}=S_{A_{1} M_{H} N_{H}}$ （на самом деле треугольники $A M_{H} N_{H}$ и $A_{1} M_{H} N_{H}$ равны, поскольку $\left.O A \perp M_{H} N_{H}\right)$. Тем самым, задача сводится к доказательству равенства площадей четырехугольника $A M_{H} A_{1} N_{H}$ и треугольника $A B C$.

Общая часть четырехугольника $A M_{H} A_{1} N_{H}$ и треугольника $A B C$ - пятиугольник $A M_{H} E F N_{H}$ (см.рис.2), поэтому достаточно понять, что $S_{E F A_{1}}=S_{E C M_{H}}+$ $+S_{F B N_{H}}$. Так как угол $A C A_{1}$ опирается на диаметр, он равен $90^{\circ}$, откуда $C A_{1} \| M_{H} H_{1}$. Далее из трапеции $M_{H} H_{1} A_{1} C$ получаем

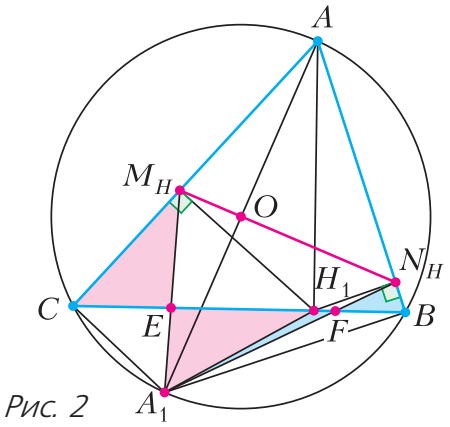

$S_{A_{1} C H_{1}}=S_{A_{1} C M_{H}}$ и $S_{E A_{1} H_{1}}=S_{E C M_{H}}$. Аналогично доказывается равенство $S_{F A_{1} H_{1}}=$ $=S_{F B N_{H}}$, и в силу равенства $S_{E F A_{1}}=$ $=S_{E H_{1} A_{1}}+S_{F H_{1} A_{1}}$ решение закончено.

Приключение второе ( точка $O$ «сбежала» с отрезка $\left.M_{H} N_{H}\right)$. Посмотрев на геометрическое решение задачи 1 , мы видим, что в доказательстве равенства $S_{A M_{H} A_{1} N_{H}}=S_{A B C}$ никак не использовалось, что точка $O$ лежит на $M_{H} N_{H}$ (это условие использовалось только в замечании о равенстве треугольников $A M_{H} N_{H}$ и $\left.A_{1} M_{H} N_{H}\right)$. Читатель может доказать равенство $S_{A M_{H} A_{1} N_{H}}=S_{A B C}$ и «формульно», снова используя формулы 1 и 2.

Если $O$ не лежит на $M_{H} N_{H}$, также в силе остаются равенства $S_{A M_{H} O}=S_{A_{1} M_{H} O}$, $S_{A N_{H} O}=S_{A_{1} N_{H} O}$ И, значит, $S_{A M_{H} O}+S_{A N_{H} O}=$ $=S_{A B C} / 2$. Таким образом, мы получили решение такой задачи:

Задача 2 (М2590). В остроугольном треугольнике $A B C$ точка $\mathrm{O}$ - чентр описанной окружности, $\mathrm{H}_{1}$ - основание высоты, проведенной из точки $A$, а $M_{H}$ и $N_{H}-$ проекции точки $H_{1}$ на $A C$ и $A B$ соответственно (рис. 3). Докажите, что лома-

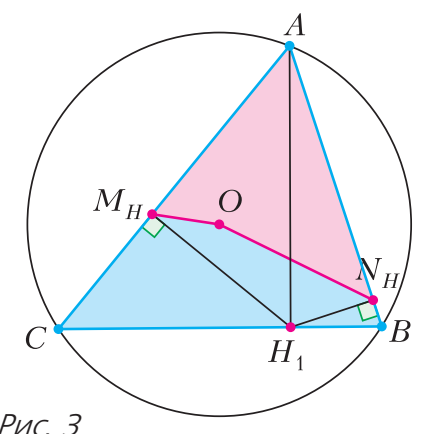

Рис. 3 
ная $M_{H} \mathrm{ON}_{H}$ делит площадь треугольника АВС пополам.

Приключение третье (диаметральная равновеликость). Удивительно, но равновеликость $S_{A M_{H} A_{1} N_{H}}=S_{A B C}$ остается в силе для бипедалей произвольной точки отрезка $B C$ (а не только для основания высоты). Получается новая задача.

Задача 3 (теорема диаметральной равновеликости). Пусть в остроугольном треугольнике АВС точка $X$ - произвольная точка отрезка $B C, X M_{X} u X N_{X}-$ бипедали этой точки, а точка $A_{1}$ диаметрально противоположна точке $A$. Докажите, что площадь четырехугольника $A M_{X} A_{1} N_{X}$ равна площади треугольника $A B C$.

Проверьте, что рассуждения из геометрического решения задачи 1 сохраняются без изменений и дают решение задачи 3.

Приведем также схему вычислительного решения задачи 3.

Обозначим через $\varphi$ угол между диаметром $A A_{1}$ и отрезком $M_{X} N_{X}$ (рис.4). Тогда $S_{A M_{X} A_{1} N_{X}}=\frac{1}{2} A A_{1} \cdot M_{X} N_{X} \sin \varphi=$ $=R \cdot M_{X} N_{X} \sin \varphi$.

Окружность с диаметром $A X$ проходит через $M_{X}, N_{X}$ и $H_{1}$. Отсюда, в частности, $M_{X} N_{X}=A X \sin \angle A$. После подстановки и «эквилибристики» $R \cdot A X \sin \angle A \sin \varphi=$ $=(R \sin \angle A)(A X \sin \varphi)$ получаем $S_{A M_{X} A_{1} N_{X}}=$ $=\frac{1}{2} B C(A X \sin \varphi)$. Поскольку $A X \sin \angle A X H_{1}=$ $=A H_{1}$, остается понять, что $\varphi$ равен $\angle A X H_{1}$

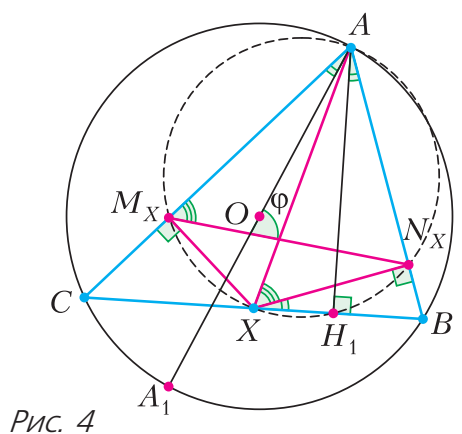

или $180^{\circ}-\angle A X H_{1}$, в зависимости от расположения точек. Несложный подсчет углов на рисунке 4 дает:

$$
\begin{gathered}
\angle A X H_{1}=\angle A X N_{X}+\angle N_{X} X H_{1}= \\
=\angle A M_{X} N_{X}+\angle N_{X} A H_{1}= \\
=\angle A M_{X} N_{X}+\angle M_{X} A A_{1}=\varphi .
\end{gathered}
$$

Приключение четвертое (изогональная равновеликость). Как и прежде, на стороне $B C$ остроугольного треугольника $A B C$ берем произвольную точку $X$ (рис.5) и проводим бипедали $X M_{X}$ и $X N_{X}$.

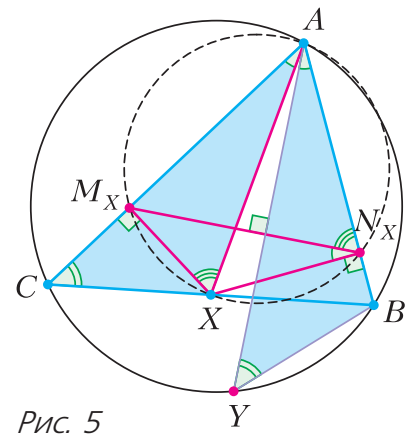

Задача 4. Точка $X$ лежит на стороне $B C$ остроугольного треугольника АBC. Через точку А проведем прямую, симметричную прямой $A X$ относительно биссектрисы угла ВАС (ее называют также изогональю для $A X)$, и пусть эта прямая пересекает описанную окружность в точке Y. Докажите, что площадь четьрехугольника $A M_{X} Y N_{X}$ равна площади треугольника $А B C$.

Отметим, что в случае, когда $A X-$ биссектриса угла $B A C, A Y$ тоже совпадает с биссектрисой, и задача 4 в этом частном случае превращается в задачу М1076, которая была придумана автором еще в 1980-х годах и вошла в вариант Международной математической олимпиады 1987 года.

В задаче 4 снова можно комбинировать как вычислительный, так и геометрический подходы. Приведем два решения.

Первое решение. Подсчет углов показывает, что $M_{X} N_{X} \perp A Y$. Действительно, $\angle A N_{X} M_{X}+\angle Y A N_{X}=\angle A X M_{X}+\angle X A M_{X}=90^{\circ}$. Далее заметим, что треугольник $A X C$ подобен (по двум углам) треугольнику $A B Y$, 
откуда $A C \cdot A B=A X \cdot A Y$. Кроме того, как мы видели ранее, $M_{X} N_{X}=A X \sin \angle A$. Отсюда получаем

$$
\begin{aligned}
S_{A M_{X} Y N_{X}}=\frac{1}{2} A Y & \cdot M_{X} N_{X}=\frac{1}{2} A Y \cdot A X \sin \angle A= \\
& =\frac{1}{2} A C \cdot A B \sin \angle A=S_{A B C} .
\end{aligned}
$$

Задача решена.

Второе решение. В силу диаметральной равновеликости (см. задачу 3), достаточно доказать равенство площадей четырехугольников $A M_{X} Y N_{X}$ и $A M_{X} A_{1} N_{X}$ (рис.6) или, что эквивалентно, равенство площадей треугольников $A_{1} M_{X} N_{X}$ и $Y M_{X} N_{X}$. Последнее следует из того, что $M_{X} A_{1} Y N_{X}-$

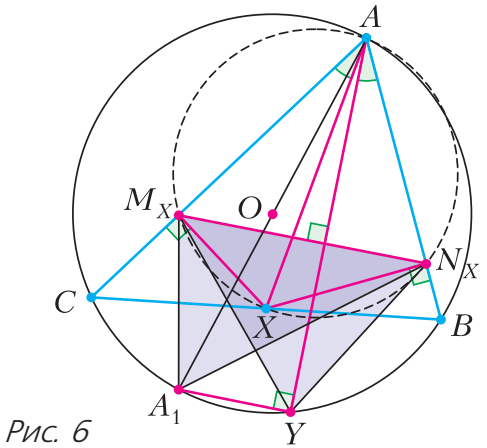

трапеция, поскольку $M_{X} N_{X} \perp A Y$ (это показано в начале первого решения) и $\angle A Y A_{1}=90^{\circ}$ (так как $A A_{1}$ - диаметр).

Bce!

\section{КОНКУРС ИМЕНИ А.П.САВИНА}

\section{ИТОГИ КОНКУРСА ИМЕНИ А.П.САВИНА 2019/20 УЧЕБНОГО ГОДА}

\section{ПОБЕДИТЕЛИ}

Лучших результатов добились школьники Бирюлин Алексей - Москва, школа 179, 7 кл.,

Дренчева Мария - Болгария, София,

Софийская математическая гимназия, 8 кл.,

Федоров Александр - Москва, школа 1514, 9 кл.,

Часовских Иван - Химки, Московская обл., школа 14, 6 кл.

\section{ПРИЗЕРЫ}

Также хороших результатов добились школьники

Сушкова Владислава - Сыктывкар, республика Коми, Физико-математический лицей-интернат, 8 кл.,

Саначев Иван - Москва, школа 1583, 4 кл.

и команды

команда математической группы Центра «Успех» Гатчинского муниципального района Ленинградской области (руководитель Павлов Сергей Павлович):
Тюков Даниил (9 кл.), Федорова Алина (9 кл.), Еронин Валерий (9 кл.), Титеева Нелли (8 кл.), Сергеев Артем (7 кл.), Кучер Анастасия (7 кл.), Валько Валентина (7 кл.), Красильников Андрей (6 кл.), Сологуб Александр (6 кл.),

команда МАОУ «Академический Лицей» города Магнитогорска (руководитель Мошкин Виталий Станиславович):

Мозговой Кирилл (9 кл.), Чернов Богдан (9 кл.), Сафронова Ольга (9 кл.), Казакова Екатерина (8 кл.), Гофштейн Лев (8 кл.), Кучкина Валерия (8 кл.), Иванова Ирина (8 кл.),

команда учеников 8 и 9 м классов школы 1329 города Москвы:

Бутрова Ирина (9 кл.), Волошин Александр (8 кл.), Галкин Савва (8 кл.), Журин Иван (8 кл.), Ильин Константин (8 кл.), Наумов Леонид (9 кл.)

\section{Поздравляем!}

Победителям и призерам будут высланы дипломы журнала «Квант» и призы от издательства МЦНМО. Помимо этого призы получат наиболее активные участники конкурса. 